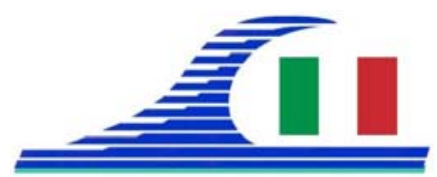

Conférence Méditerranéenne Côtière et Maritime EDITION 3, FERRARA, ITALIA (2015)

Coastal and Maritime Mediterranean Conference

Disponible en ligne - http://www.paralia.fr - Available online

\title{
Réponse des populations phytoplanctoniques (kystes et formes végétatives) face aux changements environnementaux dans les lagunes de Sidi Moussa et Oualidia, Maroc
}

\author{
Lamia DAGHOR ${ }^{1}$, Touria HSSAIDA ${ }^{1}$, Naima HAMOUMI ${ }^{2}$, Sara CHAKIR ${ }^{1}$, \\ Btissam ENEFFAH ${ }^{3}$, Mohamed FRAIKECH ${ }^{3}$, Keltoum EL BOUHMADI ${ }^{1}$
}

1. Université Hassan II de Casablanca, Faculté des Sciences Ben M’sik, B.P. 7955, Sidi Othman, Casablanca, Maroc. touria.hssaida@gmail.com

2. Université Mohammed V, Faculté des Sciences, Département de Géologie, Structure de recherche ODYSSEE, Av Ibn Batouta, BP 1014, Rabat, Maroc. naimahamoumi@yahoo.fr

3. Institut National de la Recherche Halieutique (INRH) Bd Sidi Abderrahman -Gr. Ceinture, à côté club équestre, 20050 Casablanca, Maroc.

\section{Résumé :}

L'étude qualitative et quantitative des dinoflagellés (kystes et formes végétatives) a été réalisée sur des échantillons de sédiment de surface et d'eau, prélevés au cours du printemps 2013 dans les lagunes de Oualidia et de Sidi moussa. En plus de définir le palynofaciès et de préciser les pourcentages et l'évolution spatiale de ses composantes, ce travail qui constitue une première au Maroc a permis d'identifier les paramètres environnementaux qui contrôlent :

- la concentration des kystes dans les sédiments, leur diversité spécifique ainsi que la dominance d'un groupe par rapport à un autre,

- le bloom de Lingulodinium machaerophorum dans la lagune de Oualidia et celui de Peridinium quinquecorne dans la lagune Sidi Moussa.

Mots-clés : Kystes de Dinoflagellés, Phytoplancton, Bloom, Pollution, Lagune de Oualidia, Lagune de Sidi Moussa, Maroc.

\section{Introduction}

Le littoral atlantique marocain entre El Jadida et Safi et comporte deux sites exceptionnels la lagune de Oualidia et la lagune de Sidi Moussa (Fig. 1). Ces deux écosystèmes se développent dans des dépressions allongées parallèlement à la côte atlantique. Elles sont limitées coté océan et coté continent par des cordons dunaires consolidés d'âge quaternaire et communiquent avec l'océan par 1 passe permanente au Sud et une passe temporaire plus au Nord. La lagune de Sidi Moussa est située à41 km au Sud d'El Jadida et plus précisément entre 3259',868N; 0843’,726 W et $32^{\circ} 58^{\prime}, 530 \mathrm{~N}$; 0845’,545 W. Elle a une longueur de 5,5 km, une largeur de 0,5 km et une superficie totale de $4,2 \mathrm{~km}$. La lagune de Oualidia, est située à $78 \mathrm{~km}$ au sud d’El 
Côtes méditerranéennes menacées :

Risques et défis dans le contexte du changement climatique

Jadida et plus précisément entre $32^{\circ} 45^{\prime}, 440 \mathrm{~N}$; $09^{\circ} 00^{\prime}, 870 \mathrm{~W}$ et $32^{\circ} 44^{\prime}, 950 \mathrm{~N}$; $09^{\circ} 01^{\prime}, 520 \mathrm{~W}$. Elle a une longueur de $7 \mathrm{~km}$, une largeur de $0,5 \mathrm{~km}$ et une superficie de $3 \mathrm{~km}^{2}$.

Ces deux lagunes qui sont considérées parmi les plus importantes zones humides marocaines et sont classées site d'intérêt biologique et écologique (SIBE), jouent un rôle socio-économique important dans la région (pêche, aquaculture, exploitation du sel, tourisme). En plus de l'impact lié à leur utilisation, ces deux écosystèmes sont également soumis aux rejets des zones urbaines, des activités d'agriculture et d'élevage de la plaine Doukala Aabda et des zones industrielles, dont l'unité de Jorf Lasfar située à $15 \mathrm{~km}$ en amont de la lagune de Sidi Moussa. De ce fait, elles ont suscité de nombreux travaux qui ont axés sur la géologie, la courantologie, l’hydrochimie, la systématique, l'écologie, la microbiologie et les cycles biogéochimiques (voir références in MAANAN et al., 2004 ; ZOURARAH et al., 2007; DAMSIRI et al., 2014). Les kystes de dinoflagellés n’ont jamais été étudiés et le suivi des espèces phytoplanctoniques (formes végétatives), n’a été réalisé que pour les masses d'eaux (BENNOUNA, 1999 ; NATIJ et al., 2014 ; DAMSIRI et al., 2014).

Il était donc nécessaire d'entreprendre une étude qualitative et quantitative des kystes de dinoflagellés et des formes végétatives en parallèle dans le but de préciser les facteurs qui contrôlent la concentration des kystes dans les sédiments, ainsi que leurs diversités spécifiques et leur et efflorescences. En effet, les dinoflagellés présentent un intérêt écologique indéniable, ils sont considérés comme des espèces sentinelles de la qualité

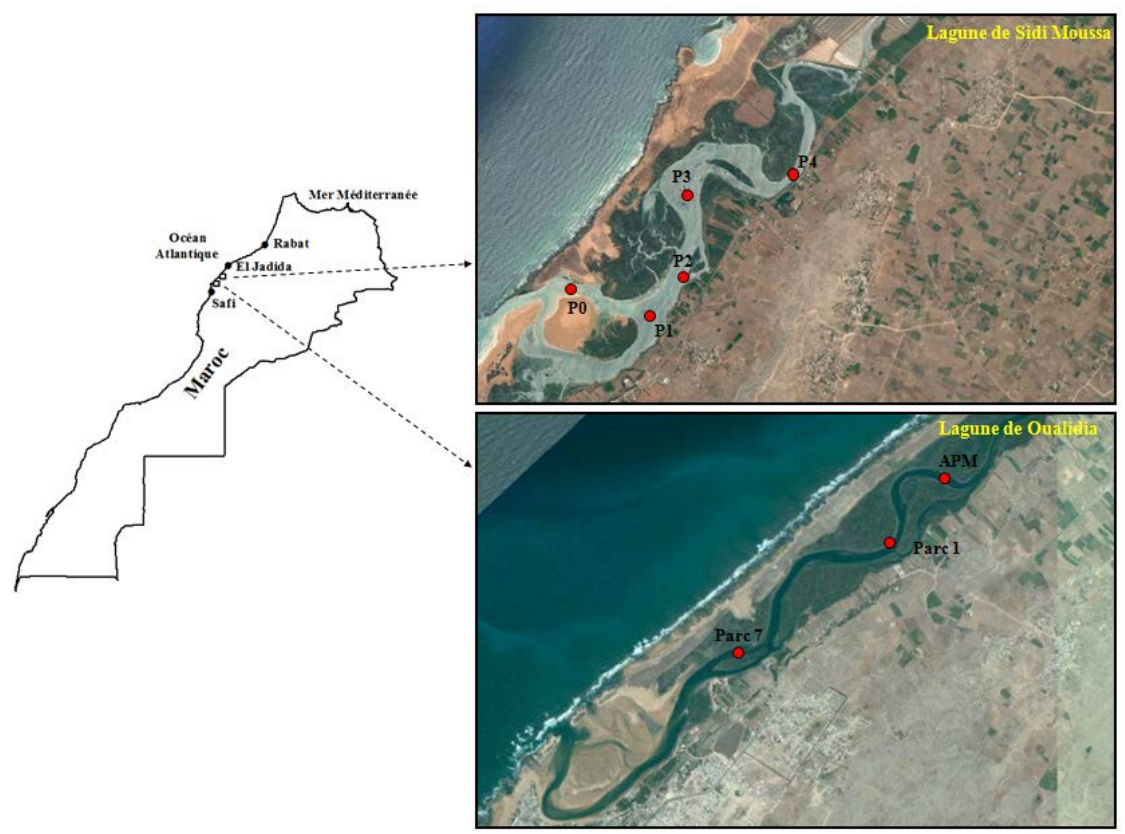

Figure 1.Vue spatiale des lagunes de Oualidia et lagune de Sidi Moussa (Images

GeoEye de Google Earth, 2014) et situation des sites d'échantillonnage. 
du littoral car ils répondent rapidement aux changements environnementaux grâce à leur cycle de vie très rapide. De plus, leurs kystes, du fait de leur extrême résistance et leur abondance dans les sédiments constituent un outil extrêmement important dans l'évaluation des environnements actuels.

\section{Matériel et méthodes}

Les dinoflagellés et les kystes des sédiments de surface, ainsi que le phytoplancton contenu dans les masses d'eau, ont fait l'objet d'une étude qualitative et quantitative. L’échantillonnage des sédiments et de l'eau a été réalisé en parallèle avec les mesures des paramètres physico chimiques de l'eau (tableau 1) au cours printemps 2013. Les sites d'échantillonnage: Parc 7 (3244',950N-0901',520W), Parc 1 (3245',440N0900',870W)1 et Ancien Parc Marost (A.P.M, 3245'33.13'’'N-900'43.27'’W) dans la lagune de Oualidia et Point 0 (32 58'49.97'’ $\mathrm{N}-8^{\circ} 44^{\prime} 47.18$ '’ $\left.\mathrm{W}\right)$, Poin1

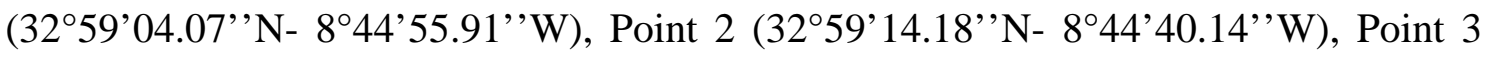

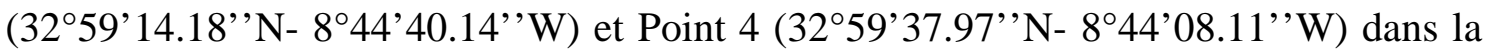
lagune de Sidi Moussa (figure 1), ont été choisis en tenant compte d'une part, de l'influence marine et des résurgences d'eaux douce et d'autre part, de la possibilité d'accéder aux sites quel que soit la marée (haute ou basse).

Le traitement des échantillons a été effectué dans le laboratoire de l'Institut National des Recherches Halieutiques (INRH) de Casa, selon la méthode classique des sédiments riche en matière organique.

Tableau 1. Paramètres physico chimiques des stations étudiées

\begin{tabular}{|c|c|c|c|c|}
\hline & $\begin{array}{l}\text { Points } \\
\text { de prélèvements }\end{array}$ & Température & $p H$ & Salinité \\
\hline \multirow{5}{*}{$\begin{array}{l}\text { Lagune de } \\
\text { Sidi Moussa }\end{array}$} & $P 0$ & 16,2 & 7,7 & 35,7 \\
\hline & $P 1$ & 17,3 & 7,8 & 33,0 \\
\hline & $P 2$ & 17,3 & 7,8 & 34,7 \\
\hline & P3 & 19,0 & 7,7 & 30,9 \\
\hline & P4 & 19,2 & 7,7 & 29,1 \\
\hline \multirow{3}{*}{$\begin{array}{l}\text { Lagune } \\
\text { de Oualidia }\end{array}$} & Parc 1 & 22,6 & 7,45 & 32 \\
\hline & Parc 7 & 20,7 & 7,89 & 25,8 \\
\hline & A.P.M & 22,7 & 7,42 & 20,3 \\
\hline
\end{tabular}

\section{Résultats}

Le palynofaciès identifié dans les sédiments de surface des deux lagunes est constitué par des kystes de dinoflagellés, des spores et pollen, des basales de foraminifères chitineux et des algues, principalement des algues vertes autochtones. Les kystes de dinoflagellés prédominent avec des pourcentages variant de 69,7\% (Parc 1 à Oualidia) à 90, 47\% (Point1 à Sidi Moussa). Le pourcentage des basales de foraminifères chitineux diminuent en allant de la passe vers le fond. Dans la lagune de Oualidia, il passe de 
Côtes méditerranéennes menacées :

Risques et défis dans le contexte du changement climatique

19,5\% (Parc 7) à 17,3\% (Parc 1) et 6,3\% (APM) et dans la lagune de Sidi Moussa, il passe de 19, 04\% (P0) à 14, 28\% dans les autres stations. Enfin, la composante «Autres » constituée par les œufs de Copépodes, les Acritarches (genre Halodinium) et les algues (surtout les algues vertes) ne dépasse pas $8 \%$ dans la lagune de Oualidia et 6,1\% dans la lagune de Sidi Moussa. Ces pourcentages et la faible richesse spécifique, indiquent un milieu restreint avec une salinité faible et une abondance des éléments nutritifs favorisée par les apports fluviaux. Cette hypothèse est confirmée par la présence du genre Halodinium, qui serait liée à ces conditions particulières (HEAD, 1993).

De plus, les associations de kystes de dinoflagellés sont dominées par l'espèce Lingulodinium machaerophorum kyste de l'espèce Lingulodinium polyedrum dont les pourcentages varient entre $70,83 \%$ et $90,47 \%$ dans la lagune de Sidi Moussa et entre $72,05 \%$ et $85,86 \%$ dans la lagune de Oualidia. La présence permanente de ce kyste, caractéristique des environnements lagunaires, témoigne d'une certaine stabilité du milieu (MORZADEC-KERFOURN, 1976 \& 1977), qui est susceptible d'être perturbée par l'augmentation de la salinité (en été) (Tableau 1) ou l'augmentation des produits azotés (rejets agricoles), provoquant ainsi des blooms ou des efflorescences, tels le bloom au niveau de la lagune de Oualidia en 1999 (BENNOUNA et al., 2002).

Cette espèce est accompagnée par deux autres kystes autotrophes, considérés comme ubiquistes vis à vis de la température: Spiniferites ramosus et Operculodinium centrocarpum qui ne dépassent pas les $12 \%$ dans la lagune de Oualidia, mais sont bien représentés, surtout le genre Spiniferites dans la lagune de Sidi Moussa, avec un taux de 28,32\%. Elle est également accompagnée par une association de kystes issus de dinoflagellés appartenant à la famille des Peridiniaceae considérés comme hétérotrophes: Selenopemphix quanta, Votadinium sp, Quinquecuspis concreta, Trinovantedinium sp, Lejeunecysta sp. Polykrikos spp.

Par ailleurs, les kystes hétérotrophes prédominent sur les kystes autotrophes, ils sont bien représentés avec des pourcentages de $40 \%$ en général, mais pouvant atteindre $42 \%$ (Point 4, lagune de Sidi Moussa). Cette prédominance pourrait s’expliquer par : (i), une eutrophisation du milieu (MATSUOKA, 1992), (ii) une pollution industrielle (SRETRE et al., 1997) et (iii)la disponibilité des nutriments ou la proximité du continent (MUDIE \& ROCHON, 2000 ; RADI, 2008).

Enfin, les dinoflagellés (formes végétatives) montrent une prolifération de l'espèce Peridinium quinquecorne, dans la lagune de Sidi Moussa (Point 3), avec une valeur maximale de 3,2 $10^{5} \mathrm{C} / \mathrm{l}$. Cette prolifération pourrait être liée à l'eutrophisation de la lagune induite par l'enrichissement du milieu en nitrites et orthophosphates, probablement en relation avec les rejets des installations industrielles de Jorf Lasfar 
Mediterranean coasts at threat:

Hazards and challenges in the context of climate variability

\section{Conclusions}

L'étude qualitative et quantitative des kystes et des formes végétatives de dinoflagellés en parallèle, dans les lagunes de Oualidia et de Sidi moussa, constitue une première au Maroc. En plus d’identifier le palynofaciès et de préciser les pourcentages et l'évolution spatiale de ses composantes, elle a permis de mettre en évidence :

- la dominance du kyste Lingulodinium machaerophorum dans les deux lagunes et dans tous les sites de prélèvement, dont les blooms fréquents sont à l'origine de l'eutrophisation;

- la dominance des kystes hétérotrophes sur les autotrophes dans les deux lagunes, qui indique l'eutrophisation du milieu et la pollution industrielle;

- la prolifération de l'espèce Péridinium quinquicorne dans la lagune de Sidi Moussa en rapport avec la richesse du milieu en nitrates et orthophosphates.

\section{Références bibliographiques}

BENNOUNA A. (1999). Etude du phytoplancton du complexe lagunaire Oualidia-Sidi Moussa. Thèse de 3ème Cycle, Université Chouaib Doukkali, Faculté des Sciences El Jadida, $149 \mathrm{p}$.

BENNOUNA A., BERLAND B., EL ATTAR J., ASSOBHEI O. (2002). Eau colorée à Lingulodiniumpolyedrum (Stein) Dodge, dans une zone aquacole du littoral du Doukkala (Atlantique marocain). Oceanologica Acta, Vol. 25, pp 159-170. http://dx.doi.org/10.1016/S0399-1784(02)01191-X

DAMSIRI Z., NATIJ L., KHALIL K., LOUDIKI M., RABOUILLE C., ETTAHIRI O., BOUGADIR B., ELKALAY K. (2014).Spatio-temporal nutrients variability in the Oualidia lagoon (Atlantic Moroccan coast). International Journal of Advanced Research, Vol. 2, pp 609-618.

HEAD H.J. (1993). Dinoflagellates, sporomorphs, and other palynomorphs from the Upper Pliocene St. Earth Beds of Cornwall, southwestern England.Paleontol. Soc. Mem., Vol. 31, pp 1-62.

MAANAN M., ZOURARAH B., CARRUESCO C., AAJANE A., NAUD J. (2004). The distribution of heavy metals in the Sidimoussa lagoon sediments (Atlantic moroccan coast).Journal of African Sciences, Vol. 39, pp 473-483. http://dx.doi.org/10.1016/j.jafrearsci.2004.07.017

MATSUOKA K. (1992).Species diversity of modern dinoflagellate cysts in surface sediments around the Japanese Islands. In Head, M. J. and Wrenn, J. H. (eds), Neogene and Quaternary Dinoflagellate Cysts and Acritarchs. A.A.S.P. Foundation, Dallas, TX, pp 33-53.

MORZADEC-KERFOURN M.T. (1976).La signification écologique des dinoflagellés et leur intérêt pour l'étude des variations du niveau marin. Revue Micropaléontologie,Vol. 18(4), pp 229-235. 
Côtes méditerranéennes menacées :

Risques et défis dans le contexte du changement climatique

MORZADEC-KERFOURN M.T. (1977). Les kystes de dinoflagellés dans les sédiments récents le long des côtes bretonnes. Revue Micropaléontologie, Vol 20(3), pp 157-166. MUDIE P.J., ROCHON A. (2001).Distribution of dino£agellate cysts in the Canadian Arctic marine region.Jounal of Quaternary Science, Vol. 16, pp 603-62. http://dx.doi.org/10.1002/jqs.658

NATIJ L., DAMSIRI Z., KHALIL K., LOUDIKI M., ETTAHIRI O., ELKALAY K. (2014). Phytoplankton abundance and diversity in the coastal waters of Oualidia lagoon, south Moroccan Atlantic in relation to environmental variables. International Journal of Advanced Research, Vol. 2, pp 1022-1032.

RADI T. (2008). Les kystes de dinoflagellés dans les sédiments de surface du pacifique du nord-est : distribution et potentiel comme proxy de paléoproductivité. Thèse Université du Québec à Montréal, Doctorat en sciences de l'environnement, 299 p.

SRETRE M.M.L., DALE B., ABDULLAH M.I., SRETRE G.P.O. (1997). Dinoflagellate cysts as potential indicators of industrial pollution in a Norwegian fjord. Marine Environmental Research, Vol. 44, pp 167-189.http://dx.doi.org/10.1016/S01411136(96)00109-2

ZOURARAH B., MAANAN M., CARRUESCO C, AAJANE A, MEHDI K., CONCEICAO F. M. (2007). Fifty-years sedimentary record of heavy metal pollution in the lagoon of Oualidia (Moroccan Atlantic coast). Estuaine coastal and shelf science, Vol. 72, pp 359-369.

http://dx.doi.org/10.1016/j.ecss.2006.11.007 